\title{
Evaluation of dynamic measurement uncertainty in the time domain in the application to high speed rotating machinery
}

\author{
Oleksandr M. Vasilevskyi ${ }^{1,}{ }^{*}$, Pavlo I. Kulakov ${ }^{1}$, Kostyantyn V. Ovchynnykov ${ }^{1}$, and Volodymyr M. Didych ${ }^{2}$ \\ ${ }^{1}$ Department of Metrology and Industrial Automation, Vinnytsia National Technical University, 95 Khmelnitskoye Shose, \\ Vinnitsya 21021, Ukraine \\ 2 Department of Biological Physics, Medical Apparatus and Informatics, Vinnytsia National Medical University named \\ after M. I. Pirogov, 56 Pirogova Street, Vinnitsya 21018, Ukraine
}

Received: 22 April 2017 / Accepted: 13 September 2017

\begin{abstract}
This article proposes a spectral method of assessing the dynamic uncertainty of measurement devices, that allows the investigation of the accuracy of measurement in dynamic operating conditions in the frequency domain and the evaluation of the amplitude values of dynamic uncertainty based on input signal frequency characteristics and spectral function. The results were tested when evaluating the dynamic uncertainty in measurements of the vibration acceleration of an engine. It was established that the maximum value of the dynamic uncertainty of the measurement of the vibration acceleration stands at $0.14 \mathrm{~m} / \mathrm{s}^{2}$ for an observation time of $300 \mathrm{~s}$ and with a nominal value of the signal of vibration acceleration of $2.34 \mathrm{~m} / \mathrm{s}^{2}$ at a frequency of $6 \mathrm{kHz}$, which in relative units is $6 \%$.
\end{abstract}

\section{Introduction}

Conducting experiments using measuring devices (MD) under dynamic conditions is becoming increasingly widespread in many areas including scientific research, technology, manufacturing industry, commerce, and medicine. Dynamic measurements are related primarily to the study of the regularities in the passage of physical processes in the subjects under study. Therefore, the role of such measurements is particularly significant, firstly, in the fields of science related to the study of the structure of matter, the analysis and synthesis of new substances and materials, the study of objects under experimental conditions. Secondly, it is important in the fields of technology, manufacturing and medicine, which are characterized by the creation of new technological processes and the testing of new MDs, by taking the concept of representation of the quality of measurements into account.

When compiling a report on the results of dynamic measurements, it is necessary to demonstrate quantitative values of the quality of measurements so that their reliability can be correctly assessed [1-11]. Without such values, the results of dynamic measurements cannot be compared, neither with each other nor with reference

* Corresponding author: o.vasilevskyi@gmail.com values. Therefore, it is necessary to propose methods for estimating the quality characteristics of dynamic measurements. In this case, it is necessary to take into account the fact that during dynamic measurements a transient mode of operation of the MD will also be present at some stage, during which the signal from the output of the measuring device changes significantly over time. These circumstances are due to the inertial properties of the MD, since they consist, as a rule, of a set of different masses and springs, capacitances and inductances, and other inertial elements that lead to the manifestation of dynamic uncertainty. The equation of the transformation of the $\mathrm{MD}$, which displays its statics properties, is unacceptable in a dynamic mode. In this case, we must go to the differential equations that describe the dynamic relationship between the output $y(t)$ and the input $x(t)$ values of the measuring devices [12-16].

In view of the above, there is a need to develop methods for estimating the uncertainty of dynamic measurements that would meet international requirements for estimating the characteristics of the quality of measurements, which is a topical scientific task in the field of metrology.

The purpose of this article is the development and mathematical description of a method for estimating the uncertainty of dynamic measurements, which would allow taking into account the inertial properties of the measuring device and the passage of the measurement signal through it. 
Table 1. Transmission functions for the most typical dynamic links.

\begin{tabular}{ll}
\hline Frequency characteristics of the MM & Typical units \\
\hline$H(j \omega)=K$ & Non-inertial (ideal measurement transducer) \\
where $K$ is the transmission coefficient & Aperiodic (temperature transducer) \\
$H(j \omega)=\frac{K}{1+j \omega \tau}$ & \\
where $\tau$ is the time constant determined & \\
by the parameters of the MM & Integrated (integrated amplification) \\
$H(j \omega)=\frac{K}{j \omega}$ & Forcing (differential amplification) \\
$H(j \omega)=K(1+j \omega \tau)$ & Delay (analog-to-digital converters) \\
$H(j \omega)=\exp (-j \omega \tau)$ & Oscillating (electromechanical transducers) \\
$H(j \omega)=\frac{K}{1+j \omega \tau_{1}-\omega^{2} \tau_{2}^{2}}=\frac{K}{1+2 j \omega \beta \tau-\omega^{2} \tau^{2}}$ &
\end{tabular}

\section{Analysis of the status of research and publications}

When composing differential equations, the input signals are written to the right-hand side, i.e., the cause of the event that brought the measuring device into action, $x(t)$, and to the left part of the differential equation, the output signal which is the response of the measuring device $y(t)[16,17]$.

To represent a differential equation in the frequency domain, the differentiation symbol for the time coordinate $d /$ $d t$ is replaced by $j \omega$ and thus the transfer function $H(j \omega)$ of the corresponding measuring transducer is obtained [14,17].

Convenient for practical use are the frequency characteristics of measuring instruments, which are given in Table $1[12,14]$.

It is also known that the concept of estimating and expressing measurement uncertainties existing in international practice [1-3], does not describe how it is possible to assess dynamic uncertainties when conducting metrological work.

The use of classical theories of determining the dynamic error in the expression of dynamic uncertainty is unacceptable, since the concept of measurement uncertainty, which is set out in an international standard [3], moves from the notion of measurement error, as such, which is not known and is not subject to being determined, as opposed to measurement uncertainty which can be evaluated. For a given measurement such a result does not have a single value, but has an infinite number of values scattered around the result. Therefore, there is a need to develop a new method for estimating dynamic uncertainty in the time domain, which could be determined without using the dynamic error used in the theory of errors.

\section{Method for estimating the uncertainty of dynamic measurements}

If the equation of the transducer under measurement can be represented in the form

$$
Y=K_{C} X
$$

where $X$ is the measured value of the physical quantity (input signal); $K_{C}$ is the coefficient of the conversion of the measuring device and $Y$ is the measurement result (output signal), then the mathematical expectation for the input signal will be equal to $M[X]$, and the mathematical expectation of the output signal will be equal to

$$
M[Y]=K_{C} M[X],
$$

where $M[Y]$ and $M[X]$ are the corresponding mathematical expectations of the output and input signals of the measuring device, respectively [18].

The spectral density of the input signal $X(t)$ has the form $[12,18]$

$$
H_{Y}(\omega)=\lim (2 T)^{-1}|X(j \omega)|^{2} \quad \text { when } T \rightarrow \infty,
$$

where $X(j \omega)$ is the Fourier image obtained by replacing the value in the operand of the image $X(s)$ by the values of $s$ for $j \omega ; T$ is the time of observation; $\omega=2 \pi f$.

The expression for the spectral density of the output signal can be represented by the expression

$$
H_{Y}(\omega)=\lim (2 T)^{-1}|Y(j \omega)|^{2} \quad \text { when } T \rightarrow \infty .
$$

The relationship between the images of the output and input values gives us an expression for the transfer function of the measuring device

$$
K_{C}(s)=\frac{Y(s)}{X(s)}=\frac{\sum_{k=0}^{m} B_{k} s^{k}}{\sum_{q=0}^{n} A_{q} s^{q}},
$$

where $Y(s), X(s)$ are the operator images of $Y(t)$ output and $X(t)$ input signals, respectively; $k, q$ are the order of the derivatives of $Y$ and $X$, respectively; $A_{q}, B_{k}$ are the coefficients of the differential equation $[12,18]$.

Therefore, we can write that

$$
H_{Y}(\omega)=\left|K_{C}(j \omega)\right|^{2} H_{X}(\omega),
$$

where $K_{C}(j \omega)$ is the frequency characteristic of the measuring transducer $[13,17]$.

The dispersion of the output signal $\sigma_{Y}^{2}$ for dynamic measurements can be defined as the square root of the integral of the spectral density of the output signal over all 
frequencies

$$
\sigma_{Y}=\pi^{-1 / 2}\left(\int_{0}^{\infty} H_{Y}(\omega) d \omega\right)^{1 / 2}
$$

Taking into account the equations (3) and (6) above, the dispersion of the output signal $\sigma_{Y}^{2} \quad(7)$ can be represented on the basis of the spectral density of the input signal and the frequency characteristic of the measuring channel used (measuring means)

$$
\sigma_{Y}=\pi^{-1 / 2}\left(T^{-1} \int_{0}^{\infty}\left|K_{C}(j \omega)\right|^{2}|X(j \omega)|^{2} d \omega\right)^{1 / 2},
$$

where $\left|K_{C}(j \omega)\right|$ is the frequency response module of the measuring device, used for dynamic measurements $[17,18]$.

The frequency response module of the measuring device is determined by the formula

$$
\left|K_{C}(j \omega)\right|=\left(a^{2}(\omega)+b^{2}(\omega)\right)^{1 / 2},
$$

where $a(\omega), b(\omega)$ are respectively, the real and imaginary parts of the frequency response $\mathrm{MD} K_{C}(j \omega)$ [18].

The spectral function of the incoming signal $X(j \omega)$ is related to its time function $X(t)$ by the Laplace expression

$$
X(j \omega)=\int_{0}^{\infty} X(t) e^{-j \omega_{0} t} d t
$$

where $\omega_{0}$ is the cyclic pulsations of the harmonic signal under study (input signal) $[17,18]$.

The cyclic pulsations $\omega_{0}$ is the pulsations of the fundamental harmonic of the investigated input signal, which passes through the measuring device (measuring channel) and is distorted due to the limited properties of the measuring channel. This frequency $\omega_{0}$ is allocated from the whole spectrum of frequencies $\omega$ to investigate the uncertainty of the measurement, which is due to the limited properties of the measuring device when passing through it a periodic signal at a certain frequency.

For a finite time interval, the infinity sign may be replace by the total observation time $T$.

To represent uncertainty of dynamic measurement as a function dependent on time $u_{D}(t)$, the Fourier expression for inverse transformation may be executed

$$
\begin{aligned}
u_{D}(t) & =\pi^{-1 / 2} \int_{0}^{\infty} \sigma_{Y} e^{j \omega t} d \omega \\
& =\pi^{-1 / 2}\left[\int_{0}^{\infty} \sigma_{Y} \cos (\omega t) d \omega+j \int_{0}^{\infty} \sigma_{Y} \sin (\omega t) d \omega\right] .
\end{aligned}
$$

Thus, the uncertainty that is introduced due to the limited properties of the measuring device used for the dynamic measurements can be estimated in the time domain, based on the model equation of the spectral function of the input signal and the frequency response of the measuring instrument used by formula (11).

Since expression (11) consists of real and imaginary parts, and in assessing the uncertainty we are interested in the amplitude value of dynamic uncertainty, expression (11) may now be written as

$$
\begin{aligned}
\left|u_{D}(t)\right|= & {\left[\left(\int_{0}^{\infty} \pi^{-1 / 2} \sigma_{Y} \cos (\omega t) d \omega\right)^{2}\right.} \\
& \left.+\left(\int_{0}^{\infty} \pi^{-1 / 2} \sigma_{Y} \sin (\omega t) d \omega\right)^{2}\right]^{1 / 2}
\end{aligned}
$$

When, for instance, prior knowledge about the amplitude of the frequency spectrum of the measurand is available in terms of a frequency function, then equation (12) can be rewritten in the form

$$
\begin{aligned}
\left|u_{D}(t)\right|= & {\left[\left(\int_{0}^{\pi F_{s}} \pi^{-1 / 2} \sigma_{Y} \cos (\omega t) d \omega\right)^{2}\right.} \\
& \left.+\left(\int_{0}^{\pi F_{s}} \pi^{-1 / 2} \sigma_{Y} \sin (\omega t) d \omega\right)^{2}\right]^{1 / 2},
\end{aligned}
$$

with $F_{s}=1 / T_{s}$ the sampling frequency.

\section{Evaluation of the uncertainty of the dynamic measurement of vibration acceleration}

In accordance with $[19,20]$, in a linear approximation, the mechanical oscillating system can be represented by one or a combination of the links of the first and second order, i.e., oscillatory link. In this case, the vibrations that are registered at the point of positioning of the accelerometer on the node of the electrospindle represent its response to the impact of the vector generating process. The differential equation describing the dynamic relationship of the input and output values of the vibration acceleration measuring transducer has the form [20]

$$
\frac{d^{2} X_{s}(t)}{d t^{2}}+2 h \frac{d X_{s}(t)}{\mathrm{d} t}+h_{k}^{2} X_{s}(t)=\frac{F_{0}}{m} \sin \left(\omega_{0} t\right),
$$

where $F(t)=F_{0} \sin \left(\omega_{0} t\right)$ is the harmonic forced power of the oscillation of the surface of the object (input value); $F_{0}$ is the force amplitude; $\omega_{0}$ is the angular frequency of forced power; $\mathrm{X}_{s}(t)$ represents the mechanical vibrations of the inertial mass; $m$ is the mass of the accelerometer; c is the damping variable; $k$ is the equivalent rigidity of the piezoelements, $h=c / 2 m$ is the damping coefficient; $h_{k}=\sqrt{k / m}$ is the critical value damping coefficient. 


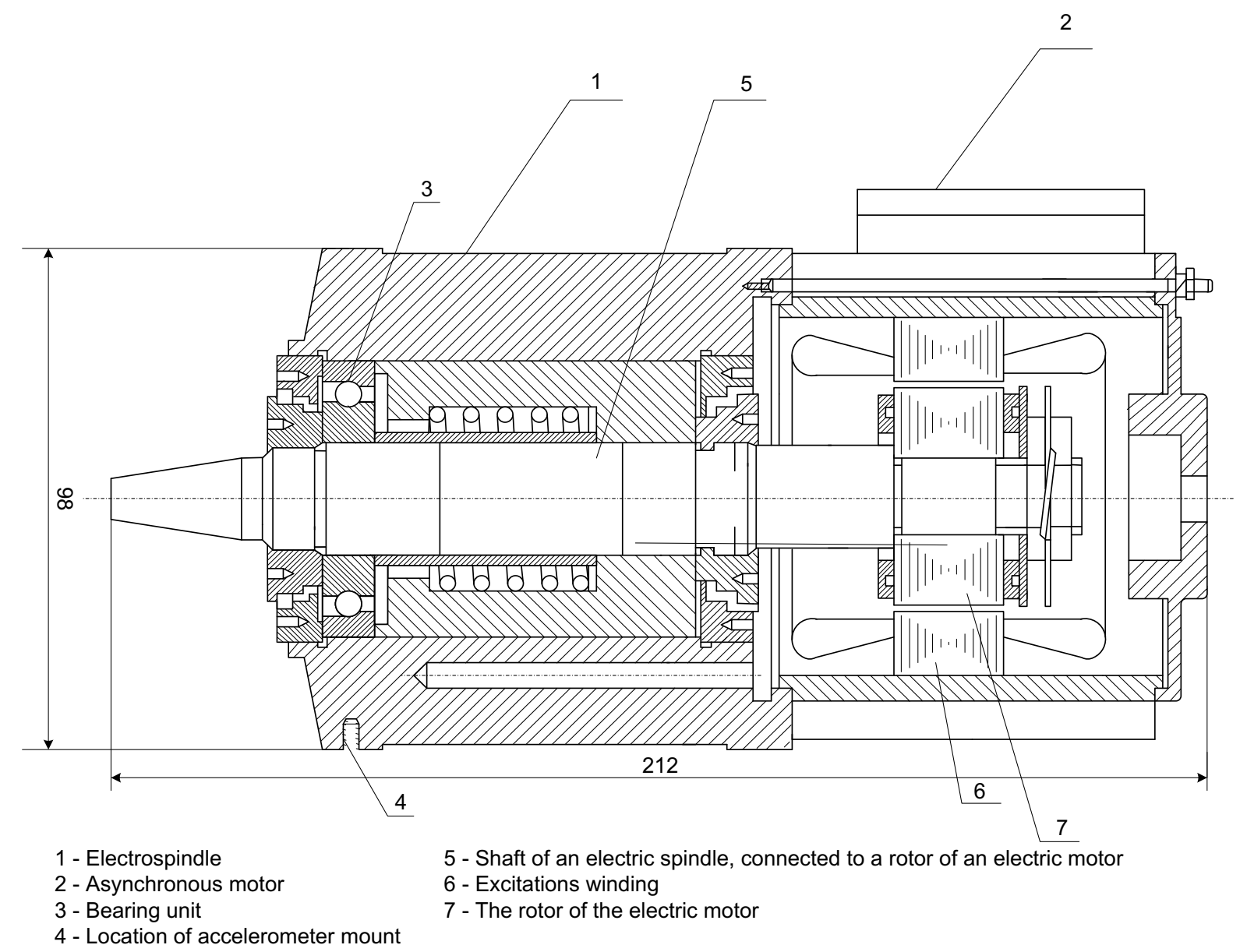

Fig. 1. Constructive drawing electrospindle.

The condition of bearings is the most important component of the technical state of the electrospindle, its serviceability and operability. At present, at least four methods for assessing the technical condition of rolling bearings are used in vibration diagnostics, which are commonly referred to as: PIK factor method; direct spectrum method; method of the envelope spectrum; shock pulse method. One of the most reliable methods of assessing the condition of bearings is the envelope spectrum method, the advantages of which are high sensitivity, informative and noise immunity. Highfrequency, the noise part of the signal changes its amplitude in time, i.e., It is modulated by some lowerfrequency signal. It turns out that it is precisely this modulating signal that contains information about the state of the bearing. The isolation and processing of this information form the basis of this method. It was experimentally established that this method yields the best results if we analyze the modulation of a nonbroadband signal obtained from the accelerometer, but beforehand we perform a bandpass filtering of the vibrational signal in the range of approximately (6$10 \mathrm{kHz}) \pm 25 \%$ [21] and analyze the modulation of this signal $[14,22]$. The choice of the frequency range $6-10 \mathrm{kHz}$ is attributed to the fact that it is in this frequency range that many nascent defects manifest themselves excitation of high-frequency vibration. It is these frequencies (first
$6 \mathrm{kHz}$, and then $10 \mathrm{kHz}$ ) that will be used as the minimum and maximum input harmonic signals $\omega_{0}$, which enters into the equation (10).

A sudden change in vibration of $25 \%$ of the specified frequency range is considered significant, which leads to the appearance of defects [21].

Therefore, we will investigate the uncertainty of the dynamic measurement of vibration acceleration with a minimum incoming frequency of the measuring signal of $6 \mathrm{kHz}$, and then the measurement uncertainty at the maximum incoming frequency is $10 \mathrm{kHz}$.

The accelerometer is located on the electrospindle of the engine and performs spot metering of the vibration in the frequency range from $6 \mathrm{kHz}$ to $10 \mathrm{kHz}$, which is created by the operation of the roller bearings (Fig. 1).

The electrospindle is a mechanical system consisting of a metal spindle body, an mounting system to asynchronous motor, rolling bearings and a shaft that is mechanically connected to the motor rotor and designed for operation at ultrahigh angular speeds (Fig. 1). It must provide a high peak power and constant action power, maximum radial and axial stiffness, maximum rotation speed, high demands on accuracy and performance. The use of electrospindles with an integrated motor for high-speed machining is possible for a number of practical applications where the required 


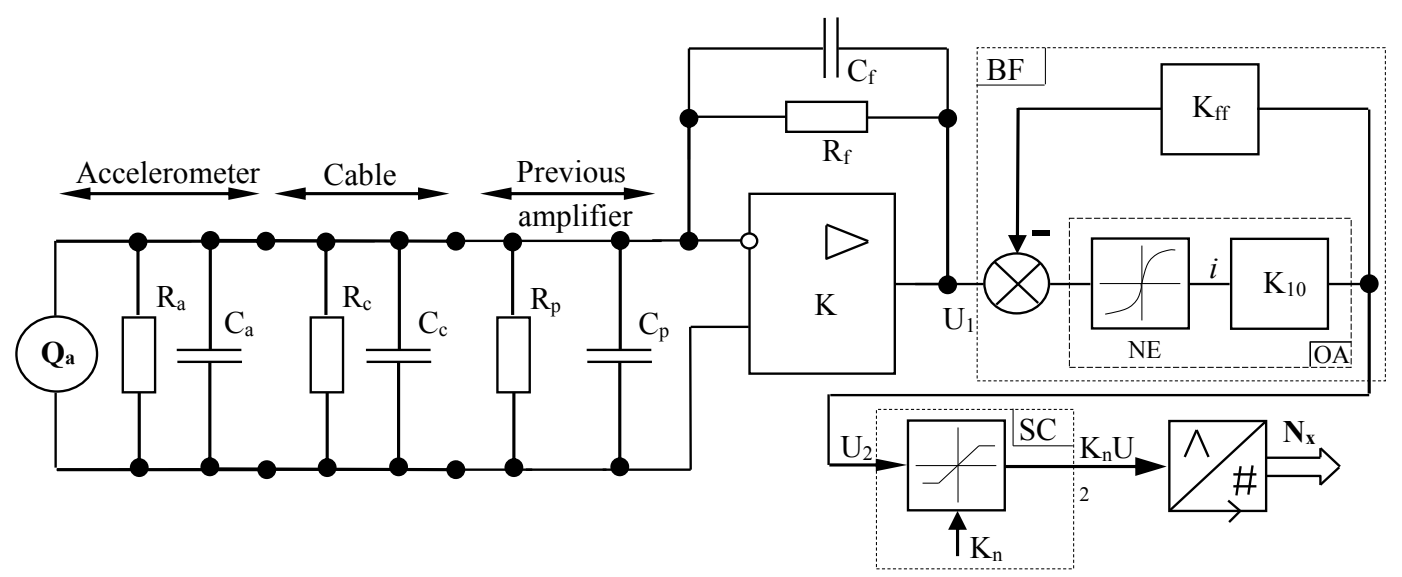

Fig. 2. Equivalent circuit measuring channel of vibration.

Table 2. Uncertainty budget of the constituent elements of the measuring channel of vibration acceleration.

\begin{tabular}{llll}
\hline Elements & $\begin{array}{l}\text { Value of relative } \\
\text { uncertainty, } \%\end{array}$ & $\begin{array}{l}\text { The expanded uncertainty } \\
\text { (coverage factor 1.96 at } \\
\text { confidence level 95\%), \% }\end{array}$ & Distribution \\
\hline Accelerometer & 0.3 & 0.59 & Uniform (rectangular) \\
Preliminary charge amplifier & 0.02 & 0.04 & Uniform (rectangular) \\
Bandpass filter & 0.2 & 0.39 & Uniform (rectangular) \\
Scale converter & 0.01 & 0.02 & Uniform (rectangular) \\
ADC & 0.005 & 0.01 & Uniform (rectangular) \\
Background noise & $2.14 \times 10^{-6}$ & $4.19 \times 10^{-6}$ & Normal \\
The combined standard & 0.36 & 0.71 & Normal \\
uncertainty $\left(\tilde{u}_{s}\right)$ & & & \\
Mean value & $2.34 \mathrm{~m} / \mathrm{s}^{2}$ & &
\end{tabular}

spindle speed can be $15000 \mathrm{rpm}$ (e.g., in the processing of diamonds or CNC (computer numerical control) machining center system).

One of the most critical components of any high-speed electrospindle is the bearing system. The electrospindle should have high speeds of rotation, transfer the necessary torque and power to the tool, have good load capacity and a long service life. Bearings must meet all these requirements.

To ensure trouble-free operation, regular maintenance and diagnosis of bearing assemblies are necessary to determine the incipient defects. These defects are determined at frequencies from 6 to $10 \mathrm{kHz}$. Therefore, the measurement of vibration in this frequency range is an actual scientific task, which allows to determine the incipient defects at early stages and to perform maintenance of the bearing units according to actual need.

To study the dynamic characteristics of measuring device that are used for dynamic measurements, one must resort to differential equations describing the dynamic relationship between output and input quantities. Therefore, the frequency characteristics of measuring devices are very convenient for compiling a model equation for dynamic measurements and estimating uncertainty, which is caused by the limited properties of measuring devices in the dynamic mode of operation, with sinusoidal input signals. In addition, in the frequency domain, it is possible to investigate a complex sinusoidal signal, which is vibration.

The equivalent circuit of the measuring channel of vibration acceleration shown in Figure 2.

Figure 2 is designated: $Q_{a}$ - electric charge; $R_{a}-$ accelerometer resistance; $\mathrm{C}_{\mathrm{a}}-$ accelerometer capacity; $R_{\mathrm{c}}$ - resistance of the connecting cable; $\mathrm{C}_{\mathrm{c}}$ - capacity of the connecting cable; $R_{\mathrm{p}}$ - resistance of the input circuit of the preamplifier; $\mathrm{C}_{\mathrm{p}}$ - capacity of the input circuit of the preamplifier; $R_{f}$ - resistance of the feedback loop; $\mathrm{C}_{f}-$ capacity of the feedback loop; $K$ - preamplifier; $U_{1}-$ output voltage of preamplifier; $\mathrm{BF}$ - bandpass filter; NE - non-linear element; $K_{10}$ - transfer coefficient BF; $K_{\mathrm{ff}}-$ feedback factor BF; $\mathrm{OA}$ - operational amplifier; $\mathrm{SC}$ scale converter; $K_{n}-$ amplification factor SC; $N_{x}$ - code analog to digital converter.

The uncertainty budget of the constituent elements of the measuring channel of the acceleration in relative units is shown in Table 2 [22-26]. On the basis of an experimental 


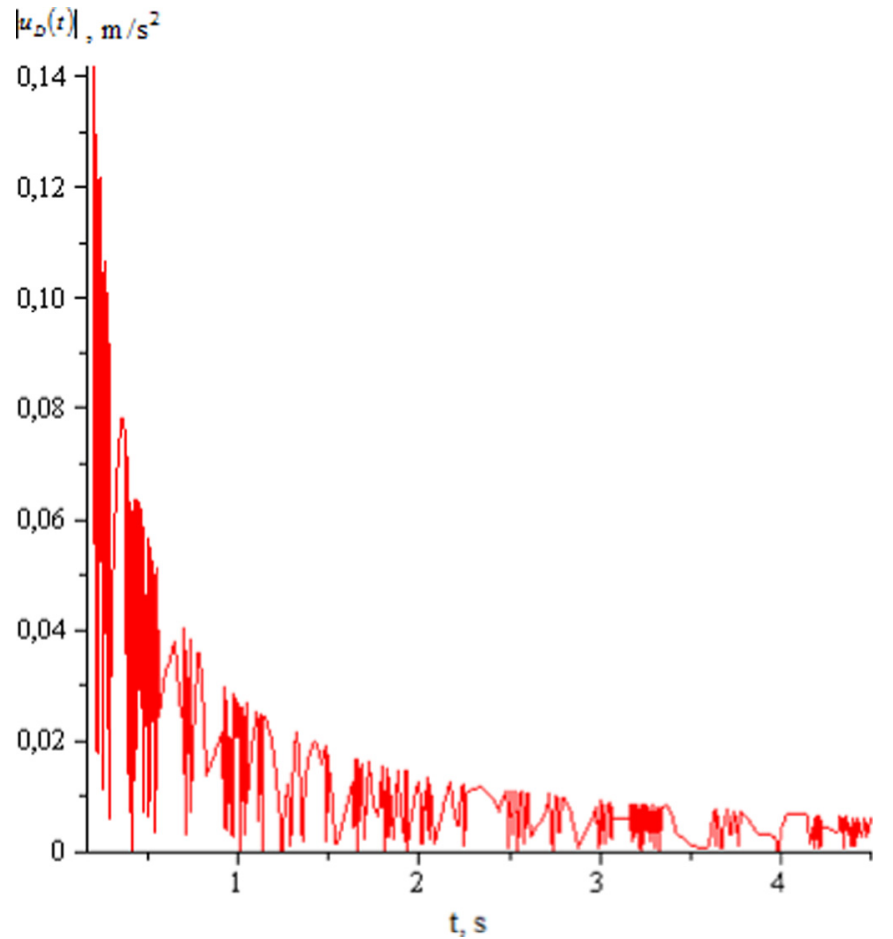

Fig. 3. Uncertainty of dynamic measurement of vibration acceleration at a frequency of $6 \mathrm{kHz}$ and observation time of $300 \mathrm{~s}$.

study of the uncertainty budget of the measurement channel of the vibration acceleration (Tab. 2), a the relative value of the combined standard uncertainty was calculated without taking into account the dynamic component of the uncertainty, which is $0.36 \%$ [26].

The transfer function of the measuring device will take the form of

$$
H(s)=\frac{K_{M M}}{s^{2}+2 h s+h_{k}^{2}},
$$

where $K_{M M}$ is the coefficient of proportionality of the measuring channel of vibration acceleration.

Turning to the domains of frequency and separating the real and imaginary parts, we obtain an expression for the module of the frequency characteristics of the measuring device for vibration acceleration

$$
\begin{aligned}
\left|K_{C}(j \omega)\right| & =\left|\frac{K_{M M}}{(j \omega)^{2}+2 h(j \omega)+h_{k}^{2}}\right| \\
& =\left\lceil\frac{K_{M M}}{\omega^{4}-2 \omega^{2} h_{k}^{2}+4 \omega^{2} h^{2}+h_{k}^{4}}\right]^{1 / 2} .
\end{aligned}
$$

The input signal $F_{0} m^{-1} \sin \left(\omega_{0} t\right)$ of vibration acceleration has the form of

$$
X(j \omega)=j \omega_{0} F_{0}\left(\omega_{0}^{2}+(j \omega)^{2}\right)^{-1} m^{-1},
$$

where $\omega_{0}$ is the frequency input vibration acceleration, which ranges from 6 to $10 \mathrm{kHz}$ that is, with a minimum value of 18849.5 and the maximum value is 31415.9 radians/second.

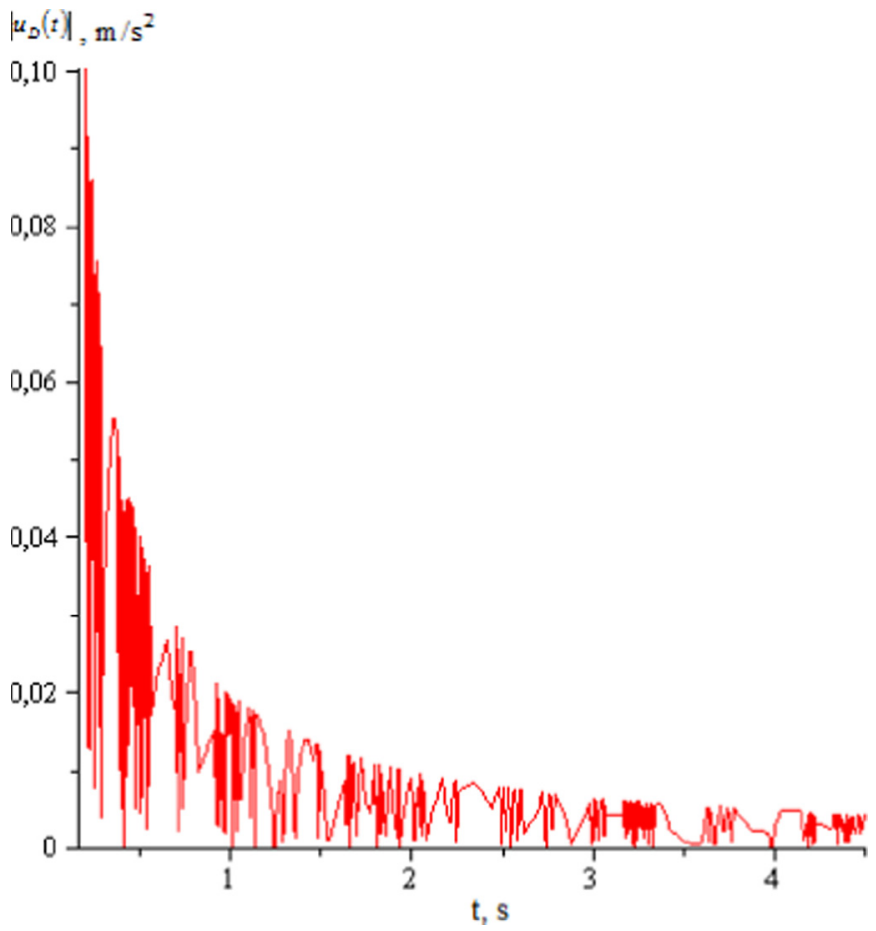

Fig. 4. Uncertainty of dynamic measurement of vibration acceleration at a frequency of $6 \mathrm{kHz}$ and observation time of $600 \mathrm{~s}$.

The module image of the input vibration acceleration is written as

$$
|X(j \omega)|=\omega_{0} F_{0}\left(\omega_{0}^{2}-\omega^{2}\right)^{-1} m^{-1} .
$$

From source literature [20,22-25], it is known that the amplitude of forced harmonic power $F_{0}$ is $3 \times 10^{-4} \mathrm{~m}$. The mass of the accelerometer is $m=4 \times 10^{-2} \mathrm{~kg}$. The damping variable for the piezoelectric accelerometers is equal to 0.5 , equivalent rigidity of the piezoelements is $k=2$, and the minimum observation time $T=300 \mathrm{~s}$. The proportionality factor or gain $K_{M M}$ of the measuring channel of the vibration acceleration is $10^{5}$.

Substituting the resulting values of the module of the frequency characteristics (16) and the image of the input signal (18) in equation (8), we obtain an expression for the evaluation of the uncertainty of dynamic measurement of vibration acceleration in the spectral area

$$
\sigma_{Y}=\pi^{-1 / 2}\left(T^{-1} \int_{0}^{\infty} \frac{K_{M M} \omega_{0}^{2} F_{0}^{2}\left(\omega_{0}^{2}-\omega^{2}\right)^{-2} m^{-2}}{\omega^{4}-2 \omega^{2} h_{k}^{2}+4 \omega^{2} h^{2}+h_{k}^{4}} d \omega\right)^{1 / 2}
$$

To represent the characteristics of the changes in the uncertainty in the dynamic measurement vibration acceleration in the time domain, which is caused by the inertial properties of the measuring transducer in its dynamic mode we must express a Fourier expression for inverse transformation in the form of (11).

Since expression (11) consists of real and imaginary parts, and in assessing the uncertainty we are interested in the amplitude value of dynamic uncertainty, expression 


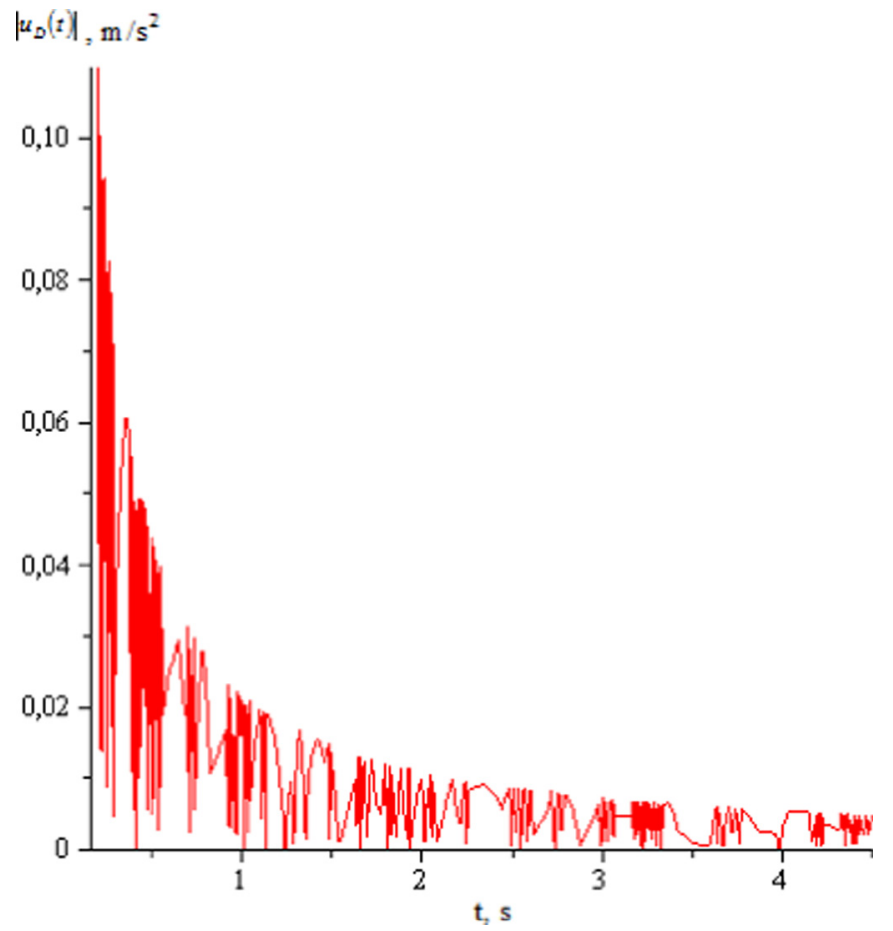

Fig. 5. Uncertainty of dynamic measurement of vibration acceleration at a frequency of $10 \mathrm{kHz}$ and observation time of $300 \mathrm{~s}$.

(11) may now be written as

$\left|u_{D}(t)\right|=\left[\pi^{-1} \int_{0}^{\pi F_{s}}\left(\sigma_{Y} \cos (\omega t)\right)^{2} d \omega+\pi^{-1} \int_{0}^{\pi F_{s}}\left(\sigma_{Y} \sin (\omega t)\right)^{2} d \omega\right]$

For the solution of equation (20) in the light of equation (19) we used the Maple 12 mathematical package. At the minimum frequency of the input signal of the vibration acceleration of $6 \mathrm{kHz}$, and with an observation time of $300 \mathrm{~s}$, the value of dynamic uncertainty is $0.14 \mathrm{~m} / \mathrm{s}^{2}$ (Fig. 3). If the observation period increased to $600 \mathrm{~s}$ at a frequency of input signal of the vibration acceleration of $6 \mathrm{kHz}$, the value of dynamic uncertainty is reduced to $0.1 \mathrm{~m} / \mathrm{s}^{2}$ (Fig. 4). The nominal value of the signal for vibration acceleration of the bearings of the electrospindle of the motor is $2.34 \mathrm{~m} / \mathrm{s}^{2}$. Characteristics of the change of dynamic uncertainty of the measurement of vibration acceleration depending on the time variable which were obtained using the Maple 12 mathematical package are presented in Figures 2 and 3, with the minimum value of the frequency of the input signal of the vibration acceleration of $6 \mathrm{kHz}$ with observation times of $300 \mathrm{~s}$ and $600 \mathrm{~s}$, are respectively.

Substituting into expressions (19) and (20) the values for the impact coefficients given above, we obtained the amplitude value of the dynamic uncertainty of the measurement of vibration acceleration, which equals $0.11 \mathrm{~m} / \mathrm{s}^{2}$ when the frequency of the input signal of the vibration acceleration is $10 \mathrm{kHz}$, and time of observation of the vibration acceleration $T=300 \mathrm{~s}$ (Fig. 5). If the time of

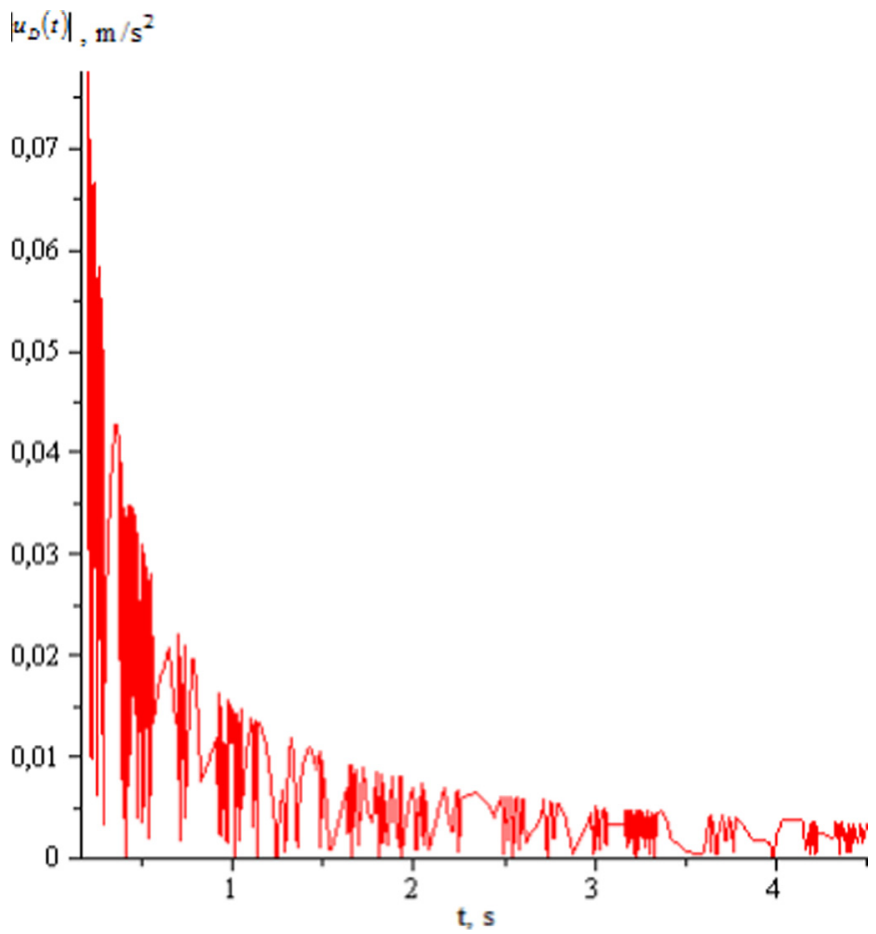

Fig. 6. Uncertainty of dynamic measurement of vibration acceleration at a frequency of $10 \mathrm{kHz}$ and observation time of $600 \mathrm{~s}$.

observation is increased to $600 \mathrm{~s}$ at the same frequency of the input signal of the vibration acceleration, the value of ${ }^{1} d^{2}$ ynamic uncertainty decreases to $0.077 \mathrm{~m} / \mathrm{s}^{2}$ (Fig. 6).

. Characteristics of the change of dynamic uncertainty of the measurement depending on the time variable which were obtained using the Maple 12 mathematical package are presented in Figures 4 and 5, with the maximum value of the frequency of the input signal of the vibration acceleration of $10 \mathrm{kHz}$ with observation times of $300 \mathrm{~s}$ and $600 \mathrm{~s}$, are respectively.

Thus, based on the proposed spectral method of evaluation of uncertainty of dynamic measurements, the evaluation of the uncertainty of dynamic measurements of vibration acceleration of roller bearings of the electrospindle of the engine was achieved. This was achieved based on mathematical models of the spectral function of the input signal of the vibration acceleration and frequency characteristics of the measurement transducer for the vibration acceleration. This resulted in obtaining the opportunity to take into account the values of dynamic uncertainties when assessing combined total uncertainty of the measurement of vibration acceleration.

To calculate the maximum relative value of the uncertainty of the dynamic measurement of vibration acceleration, we divide the obtained maximum value of dynamic uncertainty $0.14 \mathrm{~m} / \mathrm{s}^{2}$ (Fig. 3) by the nominal value of the vibration acceleration of $\bar{X}=2.34 \mathrm{~m} / \mathrm{s}^{2}$ $[14,17]$, as a result of which we obtain

$$
\tilde{u}_{D}=\frac{\left|u_{D}(t)\right|}{\bar{X}} 100 \%=\frac{0.14}{2.34} 100 \%=5.98 \% .
$$


Table 3. Uncertainty budget of the constituent elements of the measuring channel of vibration acceleration.

\begin{tabular}{lllllll}
\hline Quantity & $\begin{array}{l}\text { Mean } \\
\text { value, } \\
\mathrm{m} / \mathrm{s}^{2}\end{array}$ & $\begin{array}{l}\text { Frequency } \\
\text { of the } \\
\text { study, } \mathrm{kHz}\end{array}$ & $\begin{array}{l}\text { Observation } \\
\text { time, } \mathrm{s}\end{array}$ & $\begin{array}{l}\text { Value of } \\
\text { dynamic } \\
\text { uncertainty, } \\
\mathrm{m} / \mathrm{s}^{2}\end{array}$ & $\begin{array}{l}\text { The expanded } \\
\text { dynamic } \\
\text { uncertainty } \\
\text { (coverage factor } \\
1.96 \text { at confidence } \\
\text { level } 95 \% \text { ) } \mathrm{m} / \mathrm{s}^{2}\end{array}$ & $\begin{array}{l}\text { Value of relative } \\
\text { dynamic } \\
\text { uncertainty, } \%\end{array}$ \\
\hline $\begin{array}{l}\text { The vibration } \\
\text { acceleration }\end{array}$ & 2.34 & 6 & 300 & 0.14 & 0.27 & 5.98 \\
& 6 & 600 & 0.1 & 0.2 & 4.27 \\
& 10 & 300 & 0.11 & 0.22 & 3.7 \\
\end{tabular}

When calculating the relative value of the uncertainty of the dynamic measurement of vibration acceleration from formula (20) for the frequency of $6 \mathrm{kHz}$ and the observation time $600 \mathrm{~s}$, we obtain $4.27 \%$ (Fig. 4). At a frequency of $10 \mathrm{kHz}$ and an observation time of $300 \mathrm{~s}$ (Fig. 5), we get the relative dynamic uncertainty of the measurement of $4.7 \%$. At a frequency of $10 \mathrm{kHz}$ and an observation time of $600 \mathrm{~s}$ (Fig. 6), we get the relative dynamic uncertainty of the measurement of $3.29 \%$.

The uncertainty budget of the dynamic measurement of the vibration acceleration for the observation time of $300 \mathrm{~s}$ and $600 \mathrm{~s}$ at frequencies of 6 and $10 \mathrm{kHz}$ is shown in Table 3. The maximum value of dynamic uncertainty is $5.98 \%$ at a frequency of $6 \mathrm{kHz}$ at a observation time of $300 \mathrm{~s}$ (Tab. 3).

The relative combined uncertainty measurement of the vibration acceleration, taking into account the relative dynamic uncertainties $\tilde{u}_{D}$ (Tab. 3) and the relative combined standard uncertainty $\tilde{u}_{s}$ (Tab. 2), is calculated by the formula

$$
\tilde{u}_{C}={\sqrt{\tilde{u}_{D}}}^{2}+\tilde{u}_{s}^{2}=\sqrt{5.98^{2}+0.36^{2}}=5.99 \% .
$$

Thus, the maximum value of the relative combined uncertainty of the vibration acceleration measurement is $5.99 \%$ with an observation time of $300 \mathrm{~s}$ and a frequency of $6 \mathrm{kHz}$. At a monitoring time of $600 \mathrm{~s}$ at a frequency of $6 \mathrm{kHz}$, the combined uncertainty value is $4.29 \%$. At a frequency of $10 \mathrm{kHz}$ at a observation time of $300 \mathrm{~s}$, the combined uncertainty is $4.71 \%$ and at a observation time of $600 \mathrm{~s}$ at the same frequency of $3.31 \%$.

The permissible and optimal level of the relative combined uncertainty of the dynamic measurement of vibration is ${ }_{-20}^{+10} \%^{-20}$ of the measured vibration value $[21,27,28]$.

There are alternative methods for estimating the uncertainty of dynamic measurements, which are considered in $[28,29]$. Alternative methods for estimating the uncertainty of dynamic measurements are based on the algorithm of digital compensation filtering of the incoming signal. In them, sequences of discrete time are estimated, and the estimation of uncertainty is performed in accordance with GUM [8]. In this case, the dynamic uncertainty is calculated on the basis of an error estimate due to the imperfection of the reverse filter, using the Monte Carlo method, which is very cumbersome. But these methods do not allow to take into account the uncertainty component caused by the response of the measuring device to the frequency of the change in the incoming dynamic signal, that depends on the dynamic properties of the measuring device and the frequency spectrum of the incoming signal.

Our research resulted in establishing (Figs. 3-6), the shorter the period of time of the observation of the signal of the vibration acceleration of a moving object, roller bearing in this case, the greater the value of the dynamic uncertainty of the measurement. Therefore, to reduce the impact of dynamic uncertainty of measurements in the assessment of the results of the measurement of vibration acceleration, we must ensure an observation time of at least $600 \mathrm{~s}$.

\section{Conclusion}

The proposed spectral method of evaluating the uncertainty of dynamic measurements allows the calculation of the amplitude values of dynamic uncertainties, taking into account the international requirements for the evaluation of the quality of measurements - the concept of uncertainty. It helps to ensure the uniformity of measurements and enables comparison of the results of dynamic measurements made by different measuring devices and testing by different laboratories of leading countries. This method was tested when evaluating the dynamic uncertainty of the measurement of vibration acceleration of the roller bearing of the electrospindle of an engine, which proved its validity and effectiveness.

This method of estimating the uncertainty of dynamic measurements in the time domain is a non-statistical estimation method. This estimate is an uncertainty of type B, which can be summed up with other uncertainties to calculate the expanded uncertainty.

The proposed approach to the evaluation of dynamic uncertainty of measurement means can be used for any measurement means characterized by dynamic components of any type.

\section{References}

1. IEC GUIDE 115:2007, Application of uncertainty of measurement to conformity assessment activities in the electrotechnical sector (IEC, Switzerland, 2007) 
2. ISO/IEC 17025:2005, General requirements for the competence of testing and calibration laboratories (ISO, Switzerland, 2005)

3. ISO/IEC Guide 98-1:2009, Uncertainty of measurement Part 1: Introduction to the expression of uncertainty in measurement (ISO, Switzerland, 2009)

4. O.M. Vasilevskyi, Calibration method to assess the accuracy of measurement devices using the theory of uncertainty, Int. J. Metrol. Qual. Eng. 5, 403 (2014)

5. S. Eichstädt, C. Elster, Reliable uncertainty evaluation for ODE parameter estimation - a comparison, J. Phys.: Conf. Ser. 490, 012230 (2014)

6. O.M. Vasilevskyi, Methods of determining the recalibration interval measurement tools based on the concept of uncertainty, Tekhn. Elektrodin. 6, 81 (2014)

7. C. Elster, S. Eichstädt, A. Link, Uncertainty evaluation of dynamic measurements in line with GUM, in XIX IMEKO World Congress on Fundamental and Applied Metrology (2009)

8. Evaluation of measurement data, Guide to the expression of uncertainty in measurement, JCGM 100, GUM 1995 with minor corrections, 1st edn. (2008)

9. G. Gomah, A traceable time interval measurement with a reduced uncertainty, Int. J. Metrol. Qual. Eng. 6, 301 (2015)

10. A.B. Forbes, Uncertainty associated with form assessment in coordinate metrology, Int. J. Metrol. Qual. Eng. 4, 17 (2013)

11. O.M. Vasilevskyi, Metrological characteristics of the torque measurement of electric motors, Int. J. Metrol. Qual. Eng. 8, 7 (2017)

12. S. Eichstädt, Analysis of Dynamic Measurements - Evaluation of Dynamic Measurement Uncertainty (2012)

13. T.J. Esward, C. Elster, J.P. Hessling, Analysis of dynamic measurements: new challenges require new solutions, in Proc. of XIX IMEKO World Congress on Fundamental and Applied Metrology (2009)

14. O.M. Vasilevskyi, M. Yu. Yakovlev, P.I. Kulakov, Spectral method to evaluate the uncertainty of dynamic measurements, Tekhn. Elektrodin. 4, 72 (2017)

15. S. Eichstädt, A. Link, C. Elster, Dynamic uncertainty for compensated second-order systems, Sensors 10, 7621 (2010)

16. S. Eichstädt, C. Elster, I.M. Smith, T.J. Esward, Evaluation of dynamic measurement uncertainty - an open-source software package to bridge theory and practice, J. Sens. Sens. Syst. 6, 97 (2017)

17. O.M. Vasilevskyi, A frequency method for dynamic uncertainty evaluation of measurement during modes of dynamic operation, Int. J. Metrol. Qual. Eng. 6, 202 (2015)
18. O.M. Vasilevskyi, Evaluation of uncertainty of the results of dynamic measurements, conditioned the limited properties used the measuring instrument, in 9th International Workshop on Analysis of Dynamic Measurements, Berlin, 2016 (2016), http://mathmet.org/resources/DYNAM IC2016/Vasilevski,-Alexsandre-Dynamic-uncertainty.pdf

19. Yu. Gyzhko, M. Myslovych, Elements of the theory and practical application of systems for vibrodiagnostics of electrical machines moving parts, Techn. Electrodyn. 2, 45 (2015)

20. O.M. Vasilevskyi, P.I. Kulakov, V.M. Didych, Technique of research uncertainty dynamic measurements of vibration acceleration of rotating machines, IOSR J. Electr. Electronics Eng. (IOSR-JEEE) 11, 34 (2016), DOI:10.9790/16761105033439

21. ISO 10816-4:2009, Mechanical vibration - Evaluation of machine vibration by measurements on non-rotating parts Part 4: Gas turbine sets with fluid-film bearings (ISO, Switzerland, 2009)

22. J.T. Broch, Mechanical Vibrations and Shock Measurements, 2nd edn. (Brüel \& Kjær, 1984)

23. O.M. Vasilevskyi, Advanced mathematical model of measuring the starting torque motors, Tekhn. Elektrodin 6, 76 (2013)

24. J. Doscher, Accelerometer Design and Applications Analog Devices (1998)

25. IEC 60747-14-4:2011, Semiconductor devices - Discrete devices - Part 14-4: Semiconductor accelerometers (IEC, Switzerland, 2011)

26. O.M. Vasilevskyi, P.I. Kulakov, I.A. Dudatiev, V.M. Didych, A. Kotyra, B. Suleimenov, A. Assembay, A. Kozbekova, Vibration diagnostic system for evaluation of state interconnected electrical motors mechanical parameters, in Photonics Applications in Astronomy, Communications, Industry, and High Energy Physics Experiments 2017 (2017), Available at: http://dx.doi.org/10.1117/12.2280993

27. ISO 2954:2012, Mechanical vibration of rotating and reciprocating machinery - requirements for instruments for measuring vibration severity (ISO, Switzerland, 2012)

28. M. Kobusch, S. Eichstädt, A case study in model-based dynamic calibration of small strain gauge force transducers, ACTA IMEKO 6, 3 (2017)

29. A. Maina, I. Veldman, H. Ploug, NMISA, KEBS, BKSV trilateral vibration comparison results, ACTA IMEKO, 5, 69 (2016)

Cite this article as: Oleksandr M. Vasilevskyi, Pavlo I. Kulakov, Kostyantyn V. Ovchynnykov, Volodymyr M. Didych, Evaluation of dynamic measurement uncertainty in the time domain in the application to high speed rotating machinery, Int. J. Metrol. Qual. Eng. 8, 25 (2017) 